\title{
A NOTE ON THE REPRESENTATIONS OF NILPOTENT LIE ALGEBRAS ${ }^{1}$
}

\author{
CHARLES W. CURTIS
}

1. Introduction. H. Zassenhaus has determined ${ }^{2}$ the irreducible representations of a nilpotent Lie algebra $\&$ over an algebraically closed field $K$ of characteristic $p>0$, there being exactly one for each ordered set of characteristic values corresponding to the elements of a regular basis of $\mathbb{R}$. The question as to whether the one-to-one correspondence persists between the irreducible representations of $\&$ and ordered sets of irreducible polynomials corresponding to the elements of a regular basis of $\mathbb{Z}$ when $K$ is replaced by an arbitrary field of characteristic $p>0$ is answered affirmatively in this note. The existence of the representation belonging to a prescribed set of irreducible polynomials is proved as an application of two general results (Propositions 4 and 5 below), the first of which is a generalization of Zassenhaus' theorem ${ }^{3}$ on the existence of irreducible $\mathcal{R}-\sigma$ modules containing a given irreducible $\&$-module. Rather than dealing directly with the representation spaces, our method exploits the ideal theoretic properties of the universal associative algebra ${ }^{4}$ of $\mathfrak{R}$.

Our discussion begins with some results concerning the indecomposable representations of a nilpotent Lie algebra over an arbitrary field, and we obtain as a main result the theorem that any two irreducible constituents of such a representation are equivalent. A key step in the proof is the observation that the minimum polynomials of the representation transformations are powers of irreducible polynomials. The treatment of this point is due to Professor N. Jacobson, and was presented by him in his course at Yale in 1950-51.

The subjects considered here were brought to our attention in conversation with Professor Jacobson, and we record here our appreciation to him for many helpful suggestions concerning these matters.

\section{Indecomposable representations of nilpotent Lie algebras. We}

Presented to the Society, November 27, 1953; received by the editors December 19, 1953.

1 The research reported upon in this paper was supported in part by a Summer Research Grant from the University of Wisconsin.

${ }^{2}$ See [9] and [10] in the list of References at the end of the paper.

3 See $[9$, p. 74].

- A definition of this algebra, and the results concerning it that are needed in this paper, can be found in [1] and in [8]. 
begin with some preliminary results. Let $K$ be an arbitrary field. If $f$ is a polynomial in $K[X]$ of degree $k, f(X)=\sum_{i=0}^{k} \beta_{i} X^{i}$, then for each $j, 0 \leqq j \leqq k$, we define $f_{j}(X)=\sum_{i=j}^{k} C_{i, j} \beta_{i} X^{i-j}$. The mapping $f \rightarrow f_{j}$ is a 1.t. ${ }^{5}$ in $K[X]$. We can then establish Leibniz's rule

$$
(f g)_{j}=\sum_{i=0}^{j} f_{i} g_{j-i}
$$

by observing first, by a direct calculation, that it is valid for $f(X)$ $=X^{r}, g(X)=X^{s}$, and that it holds in $K[X]$ by linearity.

Lemma 1. If $f$ and $g$ are polynomials in $K[X]$ such that $f^{j+1} \mid g$, then $f \mid g_{i}, i=0,1, \cdots, j$.

Proof. We use induction on $j$, noting that the result is clear if $j=0$. Assume that it holds for $j-1$, and let $f^{i+1} \mid g$. Then we can write $g=h k$, where $f^{i} \mid h$ and $f \mid k$. By induction, $f \mid h_{0}, \cdots, h_{j-1}$. Then by (1), $g_{j}=\sum_{i=0}^{j} h_{i} k_{j-i}$, and $f \mid g_{j}$ as required.

Before stating the next lemma, we list a number of formulas which we shall need. Let $\Re$ be an associative ring, and let $a, d \in \Re$. Then

$$
a d^{k}=d^{k} a+C_{k, 1} d^{k-1} a^{\prime}+C_{k, 2} d^{k-2} a^{\prime \prime}+\cdots+a^{(k)},
$$

$k=1,2, \cdots$, where $a^{\prime}=[a, d]$, and $a^{(i)}$ is defined recursively by $a^{(i)}=\left[a^{(i-1)}, d\right]$. If $\Re$ has characteristic $p>0$, then we obtain from (2)

$$
a d^{p}=d^{p} a+a^{(p)}, \text { and } a d^{p^{k}}=d^{p k} a+a^{\left(p^{k}\right)}, \quad k=1,2, \cdots .
$$

On the other hand, if $\Re$ is an algebra over $K$, then another application of (2) yields

(4) $a f(d)=f(d) a+f_{1}(d) a^{\prime}+f_{2}(d) a^{\prime \prime}+\cdots+f_{k}(d) a^{(k)}, \quad f \in K[X]$.

Now let $A$ and $D$ be 1.t. in a finite-dimensional vector space $\mathfrak{M}$ over $K$. For each polynomial $f \in K[X]$, we define $\mathfrak{M}_{f, D}$ to be the subspace of $\mathfrak{M}$ consisting of those vectors $y$ that are annihilated by some power of $f(D)$. Then we obtain the following result.

LEMma 2. If $A$ and $D$ are l.t. such that $A^{(n)}=[[\cdots[A D] D] \cdots D]$ $=0$ (n D's) for some $n$, then $\mathfrak{M}_{f, D} A \subseteq \mathfrak{M}_{f, D}$, for every $f \in K[X]$.

Proof. Let $y \in \mathfrak{M}_{f, D}$, and suppose that $y(f(D))^{m}=0$. If we set $g=f^{m}, h=g^{n}=f^{m n}$, then by (4) we have

$$
A h(D)=h(D) A+h_{1}(D) A^{\prime}+\cdots+h_{n-1}(D) A^{(n-1)} .
$$

Since $g^{n} \mid h$, we have by Lemma $1, g \mid h_{i}, i=0,1, \cdots, n-1$, and by

${ }^{5}$ We shall use the abbreviation 1.t. for linear transformation. 
(5), $y A h(D)=0$, proving that $y A \in \mathfrak{M}_{f, D}$.

Proposition 1. Let $\&$ be an indecomposable Lie algebra of l.t. in a finite-dimensional vector space $\mathfrak{M}$ over $K$, and let $D$ be a l.t. such that $[R, D] \in \mathbb{R}$. If the derivation $A \rightarrow[A, D]$ in $\&$ is nilpotent, then the minimum polynomial of $D$ is a power of an irreducible polynomial.

Proof. Let $f$ be the minimum polynomial of $D$, and let $f$ $=h_{1}^{e_{1}} \cdots h_{r}^{e_{r}}$ be the prime factorization of $f$ in $K[X]$, where the $h_{i}$ are distinct irreducible polynomials. Suppose that $r>1$; then we have $\mathfrak{M}=\mathfrak{M}_{h_{1}, D} \oplus \cdots \oplus \mathfrak{M}_{h_{r}, D}$, where the $\mathfrak{M}_{h_{i}, D} \neq(0)$. On the other hand, for each $A \in \mathbb{R}, A^{(n)}=[[A, D] \cdots D]=0$ for some $n$, and Lemma 2 implies that the subspaces $\mathfrak{M}_{h_{i}, D}$ are invariant relative to $\mathfrak{R}$, contradicting our hypothesis that $\&$ is indecomposable.

Corollary. Let \& be an indecomposable nilpotent Lie algebra of l.t. in a finite-dimensional vector space. Then the minimum polynomial of every $A \in \mathbb{R}$ is a power of an irreducible polynomial.

PRoposition 2. Let $x \rightarrow U_{x}$ be a representation of finite degree of a nilpotent Lie algebra $\&$ over an infinite field $K$. If $f^{6} \operatorname{det} U_{x}=0$ for all $x \in R$, then there exists a nonzero vector $v$ in the representation space such that $v U_{x}=0$ for every $x \in$ ?.

Proof. This result has been proved by Chevalley [3] for fields of characteristic zero; we shall indicate how his proof can be modified so that it holds for an infinite field $K$ of characteristic $p>0$. Let $U(\mathfrak{R})$ be the set of 1.t. $U_{x}, x \in \mathfrak{R}$, and let $\mathfrak{M}$ be the representation space of $U$. The proof is carried out by induction on the dimension of $\mathfrak{M}$, the result being clear if the dimension is one. First we suppose that $U$ is an irreducible representation. By (3) there exists a $k$ independent of $x$ such that the $p^{k}$ th power of each element of $U(\Omega)$ is in the center of the enveloping algebra of $U(\Omega)$, and by Schur's Lemma, if $U_{x}^{p^{k}} \neq 0$, then $U_{x}^{p^{k}}$ is an automorphism of $\mathfrak{M}$, and $U_{x}$ cannot be singular. Thus $U_{x}$ is nilpotent for every $x \in \mathbb{R}$. By a theorem of Jacobson [7] the enveloping algebra $\mathfrak{A}$ of $U(\mathfrak{R})$ is a nilpotent algebra, and it follows that $\mathfrak{M A}=(0)$. Therefore $\mathfrak{M U} U(\mathbb{R})=(0)$.

The remainder of the proof is identical with the characteristic zero case, and for the sake of completeness, we shall include it. If $U$ is reducible, then we select a maximal submodule $\mathfrak{P}$ of $\mathfrak{M}$; by the first part of the proof we may assume that $\mathfrak{B} \neq(0)$. Let $U^{\prime}$ be the induced representation in $\mathfrak{M}-\mathfrak{B}$, and let $U^{\prime \prime}$ be the restriction of $U$ to $\mathfrak{B}$. Then we have $\operatorname{det} U_{x}=\operatorname{det} U_{x}^{\prime} \cdot \operatorname{det} U_{x}^{\prime \prime}=0$ for all $x \in R$. The func-

${ }^{6}$ By det $U_{x}$ we mean the determinant of a matrix belonging to $U_{x}$. 
tions $U_{x}^{\prime} \rightarrow \operatorname{det} U_{x}^{\prime}$ and $U_{x}^{\prime \prime} \rightarrow \operatorname{det} U_{x}^{\prime \prime}$ are polynomial functions on the subspaces of the algebras of 1.t. of $\mathfrak{M}-\mathfrak{B}$ and $\mathfrak{P}$ generated by the sets $U^{\prime}(\Omega)$ and $U^{\prime \prime}(\Omega)$ respectively. Since $K$ is an infinite field, one factor must vanish identically $\left[2\right.$, p. 27]. If $\operatorname{det} U_{x}^{\prime \prime}=0$ for all $x \in \Omega$, then our induction hypothesis yields the result, so we may suppose that for some $a \in R$, det $U_{a}^{\prime \prime} \neq 0$, and that det $U_{x}^{\prime}=0, x \in R$. By the first part of the proof we have $U^{\prime}(\mathfrak{R})=(0)$, so that $\mathfrak{M} U(\mathfrak{R}) \subseteq \mathfrak{B}$. Now since det $U_{a}=0$, for some $y \notin \mathfrak{B}, y U_{a}=0$. Let $\mathfrak{Y}=y U(\mathfrak{R})$. Then $\mathfrak{Y} \subseteq \mathfrak{B}$, and for each $x \in R, y U_{x} U_{a}=y U_{[x, a]}+y U_{a} U_{x}=y U_{[x, a]}$ since $y U_{a}=0$. By induction we have $y U_{x} U_{a}^{k}=y U_{[\cdots[x, a] \cdots a]}=0$ ( $k a$ 's) for sufficiently large $k$. If some $y U_{x} \neq 0$, then we can find $k$ such that $z=y U_{x} U_{a}^{k-1} \neq 0$, and $z U_{a}=0$. Since $z \in \mathfrak{P}$, this contradicts our assumption that $U_{a}^{\prime \prime}$ is nonsingular on $\mathfrak{P}$. Thus $\mathfrak{Y}=y U(\mathfrak{R})=(0)$, and the proof is complete.

THEOREM 1. Let $\mathfrak{R}$ be a nilpotent Lie algebra over an arbitrary field $K$. If $x \rightarrow U_{x}$ is an indecomposable representation of $\mathbb{R}$ of finite degree, then any two irreducible constituents of $U$ are equivalent.

Proof. Let $x \rightarrow R_{x}$ and $x \rightarrow S_{x}$ be two irreducible constituents of $U$. If $\mathfrak{B}$ is a representation space of $U$, then $R$ and $S$ must appear as representations induced upon two composition factors of $\mathfrak{B}$ arising from a single composition series of $\mathfrak{B}$. It follows that, for each $x \in L$, the minimum polynomials $h_{x}$ and $k_{x}$ of $R_{x}$ and $S_{x}$ respectively must divide the minimum polynomial of $U_{x}$, which is a power of an irreducible polynomial by Proposition 1 . Therefore the minimum polynorials $h_{x}$ and $k_{x}$ are powers of the same irreducible polynomial in $K[X]$ and, over an algebraic closure of $K$, will have identical sets of distinct roots.

If we now extend the base field to $K^{\prime}$, the algebraic closure of $K$, then $h_{x}$ and $k_{x}$ remain the minimum polynomials of the extended l.t. $R_{x}^{\prime}$ and $S_{x}^{\prime}$. Let $e$ and $f$ be the degrees (over $K^{\prime}$ ) of the representations $R^{\prime}$ and $S^{\prime}$, and let $x \rightarrow T_{x}^{\prime}$ be the Kronecker sum representation $x \rightarrow R_{x}^{\prime} \otimes 1_{f}+1_{e} \otimes\left(-\left(S_{x}^{\prime}\right)^{*}\right)$ of the representations $R^{\prime}$ and $-S^{\prime *}$, where $S^{*}$ is the transpose of $S^{\prime}$. Then by an argument given by Zassenhaus [10, p. 154], zero must appear as a characteristic value of each of the l.t. $T_{x}^{\prime}$. Therefore $\operatorname{det} T_{x}^{\prime}=0, x \in \mathfrak{R}$, and by Proposition 2, since $K^{\prime}$ is an infinite field, there exists a nonzero vector $w^{\prime} \in \mathfrak{W}^{\prime}$, where $\mathfrak{W}^{\prime}$ is a representation space for $T^{\prime}$, such that $w^{\prime} T^{\prime}(\mathbb{R})=(0)$.

The representation space of $R^{\prime}$ is $\mathfrak{B} \otimes K^{\prime}$, while that of $S^{\prime}$ is $\mathfrak{B} \otimes K^{\prime}$, where $\mathfrak{B}$ and $\mathfrak{B}$ are representation spaces of $R$ and $S$ respectively. The representation space of $T^{\prime}$ is therefore $\left(\mathfrak{B} \otimes K^{\prime}\right) \otimes\left(\mathfrak{B} \otimes K^{\prime}\right)$, which is isomorphic to $(\mathfrak{B} \otimes \mathfrak{B}) \otimes K^{\prime}$. Moreover, since $R^{\prime}=R \otimes 1$, 
and $S^{\prime}=S \otimes 1$, where 1 is the identity element of $K^{\prime}$, we have

$$
\begin{aligned}
T^{\prime} & =(R \otimes 1) \otimes 1_{f}+1_{e} \otimes\left(-S^{*} \otimes 1\right) \\
& =\left(R \otimes 1_{f}+1_{e} \otimes-S^{*}\right) \otimes 1 .
\end{aligned}
$$

Thus $T^{\prime}$ is obtained by extension of the base field from the representation $T=R \otimes 1_{f}+1_{e} \otimes-S^{*}$, and the representation space of $T^{\prime}$ is the extension by $K^{\prime}$ of the representation space $\mathfrak{B} \otimes \mathfrak{B}$ of $T$. If we write $w^{\prime}=\sum w_{i} \lambda_{i}, \lambda_{i} \in K^{\prime}$, where the $\lambda_{i}$ are linearly independent over $K$, then we have $w^{\prime} T_{x}^{\prime}=\sum\left(w_{i} T_{x}\right) \lambda_{i}=0$ for each $x \in \mathbb{R}$, and hence each vector $w_{i} T_{x}=0$. In particular, if $w_{1} \neq 0$ then $w_{1} T(R)=(0)$, and an application of Schur's Lemma yields the result that $R$ and $S$ are equivalent $[10$, p. 154].

Proposition 3. Let $\mathfrak{S}$ be an indecomposable set of l.t. in a finitedimensional space $\mathfrak{B}$, let $\mathfrak{A}$ be the associative algebra generated by the elements of $\mathfrak{S}$ and the identity l.t., and let $\mathfrak{N}$ be the radical of $\mathfrak{A}$. Then $\mathfrak{U} / \mathfrak{R}$ is simple if and only if any two of the composition factors of $\mathfrak{B}$ relative to $\subseteq$ are $\subseteq$-isomorphic.

Proof. Let $\mathfrak{B}=\mathfrak{B}_{0} \supset \mathfrak{B}_{1} \supset \ldots \supset \mathfrak{B}_{t+1}=(0)$ be a composition series of $\mathfrak{B}$ relative to $\mathfrak{S}$; then this will be a composition series of $\mathfrak{B}$ relative to $\mathfrak{A}$. Suppose first that the composition factors $\mathfrak{B}_{i}-\mathfrak{B}_{i+1}$ are $\mathfrak{S}$-isomorphic, and hence $\mathfrak{A}$-isomorphic. Then $\mathfrak{B}_{t}$ is an irreducible $\mathfrak{A}$-module such that $\mathfrak{B}_{t} \mathfrak{A} \neq(0)$, hence $\mathfrak{B}_{t} \mathfrak{N}=(0)$, and $\mathfrak{B}_{t}$ is an irreducible $\mathfrak{A} / \mathfrak{N}$ module. Suppose that for some $a \in \mathfrak{A}, \mathfrak{B}_{t} a=(0)$. Then for any $x \in \mathfrak{A}$, $\mathfrak{B}_{t} a x=(0)$. For each $i$, there exists an $\mathfrak{A}$-isomorphism $E_{i}$ of $\mathfrak{B}_{t}$ onto $\mathfrak{B}_{i}-\mathfrak{B}_{i+1}$. Then $\left(\mathfrak{B}_{i}-\mathfrak{B}_{i+1}\right) a x=\left(\mathfrak{B}_{t} E_{i}\right) a x=\left(\mathfrak{B}_{i} a x\right) E_{i}=(0), i=0,1$, $\cdots, t$, and $\mathfrak{B}_{i} a x \subseteq \mathfrak{B}_{i+1}, i=0,1, \cdots, t$. Thus $(a x)^{t+1}=0$, and since $x$ is arbitrary in $\mathfrak{A}, a \in \mathfrak{N}$. Therefore $\mathfrak{B}_{t}$ is a faithful irreducible $\mathfrak{A} / \mathfrak{N}$-module, and $\mathfrak{A} / \mathfrak{N}$ is a simple algebra.

Conversely, if $\mathfrak{A} / \mathfrak{N}$ is simple, then since $\mathfrak{A}$ contains the identity mapping, the irreducible factors of $\mathfrak{B}$ are isomorphic to minimal right ideals in $\mathfrak{A} / \mathfrak{N}$, and hence are $\mathfrak{A}$-isomorphic, and, a fortiori, $\mathfrak{S}$-isomorphic.

CoRollary. Let $x \rightarrow U_{x}$ be an indecomposable representation of a nilpotent Lie algebra $\mathfrak{R}$ over an arbitrary field $K$. Then the associative algebra generated by the transformation $U_{x}$ and the identity transformation is a primary algebra.

The proof is a direct application of Theorem 1 and Proposition 3.

3. Extensions of irreducible representations. If $\&$ is a Lie algebra over $K$, and if $\mathfrak{D}$ is a Lie algebra of derivations of $\mathfrak{R}$, then the semi- 
direct sum $\mathfrak{R}+\mathfrak{D}$ is the Lie algebra whose underlying vector space is the direct sum of the vector spaces $\mathbb{R}$ and $\mathfrak{D}$, and in which the bracket operation is defined by the formula

$$
\begin{array}{r}
{\left[x+D, x^{\prime}+D^{\prime}\right]=\left[x, x^{\prime}\right]+x D^{\prime}-x^{\prime} D+\left[D, D^{\prime}\right],} \\
x, x^{\prime} \in \mathcal{R} ; D, D^{\prime} \in \mathfrak{D} .
\end{array}
$$

Then it is well known that $\mathfrak{R}+\mathfrak{D}$ is a Lie algebra containing $\mathfrak{l}$ as an ideal.

Proposition 4. Let $\mathfrak{R}$ be a Lie algebra over an arbitrary field $K$, and let $\mathfrak{D}$ be a Lie algebra of derivations of $\mathfrak{R}$. If $x \rightarrow U_{x}$ is an irreducible representation of $\mathbb{R}$ with representation space $\mathfrak{B}$, then there exists an irreducible representation $x \rightarrow T_{x}$ of $\mathfrak{Q}+\mathfrak{D}$ with representation space $\mathfrak{W}$, and a one-to-one l.t. $S$ of $\mathfrak{B}$ into $\mathfrak{B}$ such that $U_{x} S=S T_{x}$ for all $x \in \mathbb{R}$. Moreover, if the base field has characteristic $p>0$, so that the degree of the representation $U$ is finite, a representation $T$ of finite degree can be found which satisfies the above requirements.

Proof. First suppose that $U(\mathfrak{R})=(0)$; then $\mathfrak{B}$ is one-dimensional. We set $\mathfrak{B}=\mathfrak{B}$, let $D \rightarrow D^{\prime}$ be any linear mapping of $\mathfrak{D}$ into the set of 1.t. of $\mathfrak{B}$, and let $T_{x+D}$ be the mapping $D^{\prime}$ in $\mathfrak{W}$. If we let $S$ be the identity mapping in $\mathfrak{B}$, then the requirements of Proposition 4 are easily verified.

Now let $\mathfrak{B} U(\mathfrak{R}) \neq(0)$; let $\mathfrak{A}$ be the universal associative algebra of $\mathfrak{R}$, and let $\mathfrak{H}^{\prime}$ be the universal associative algebra of $\mathfrak{R}+\mathfrak{D}$. If $x_{1}, \cdots$, $x_{n}$ is a basis for $\mathfrak{R}, D_{1}, \cdots, D_{m}$ a basis for $\mathfrak{D}$, then the standard monomials $x_{1}^{e_{1}} \cdots x_{n}^{e_{n}} D_{1}^{f_{1}} \cdots D_{m}^{f_{m}}, e_{i}, f_{i} \geqq 0$, form a basis for $\mathfrak{A}^{\prime}$. The subalgebra of $\mathfrak{H}^{\prime}$ generated by the elements $1, x_{1}, \cdots, x_{n}$ is isomorphic to $\mathfrak{A}$, and we shall identify this subalgebra with $\mathfrak{A}$. The representation $U$ can be extended in a unique way to a representation of $\mathfrak{A}$ which we shall again denote by $U$. Since $\mathfrak{B} U(\mathfrak{R}) \neq(0), \mathfrak{B}$ is a cyclic $\mathfrak{A}$-module, and is $\mathfrak{A}$-isomorphic to $\mathfrak{A}-\mathfrak{Y}$ where $\mathfrak{Y}$ is a maximal right ideal in $\mathfrak{A}$.

We prove first that the right ideal $\mathfrak{F}^{\prime}=\left\{\sum x_{i} b_{i}, x_{i} \in \mathfrak{F}, b_{i} \in \mathfrak{A}^{\prime}\right\}$ is a proper ideal in $\mathfrak{A}^{\prime}$. Every element in $\mathfrak{A}^{\prime}$ can be expressed uniquely in the form

$$
a_{0}+\sum a_{i_{1} \ldots i_{m}} D_{1}^{i_{1}} \cdots D_{m}^{i_{m}}, \quad a_{0}, a_{i_{1}} \cdots i_{m} \in \mathfrak{A},\left(i_{1}, \cdots, i_{m}\right) \neq 0 .
$$

Then $\Im^{\prime}{ }^{\prime}$ consists of all elements (6) such that the coefficients $a_{0}, a_{i_{1} \cdots i_{m}} \in \mathfrak{Y}$. If $\mathfrak{S}^{\prime} \mathfrak{Y}^{\prime}=\mathfrak{Y}^{\prime}$, then the identity element 1 can be expressed in the form (6) with coefficients in $\Im$. It then follows from considerations of degree that $a_{0}=1 \in \Im$, contrary to the fact that $\Im$ is a proper ideal in $\mathfrak{A}$. Then $\mathfrak{Y} \mathfrak{H}^{\prime}$ can be imbedded in a maximal right 
ideal $\mathfrak{Y}^{\prime}$ in $\mathfrak{A}^{\prime}$, and since $\mathfrak{Y}^{\prime} \supseteq \mathfrak{H}^{\prime} \supseteq \Im, \mathfrak{Y}^{\prime} \cap \mathfrak{U} \supseteq \Im$. But $\mathfrak{Y}^{\prime} \cap \mathfrak{A}$ is a proper right ideal in $\mathfrak{A}$, and since $\mathfrak{Y}$ is maximal, $\mathfrak{Y}^{\prime} \cap \mathfrak{A}=\mathfrak{Y}$.

Then $\mathfrak{Y}^{\prime}-\mathfrak{Y}^{\prime}$ is an irreducible $\mathfrak{I}^{\prime}$-module, and if we define $T_{x}: u+I^{\prime}$ $\rightarrow u x+I^{\prime}$ for $x$ restricted to the linear part of $\mathfrak{Y}^{\prime}$, then we obtain an irreducible representation of $\mathfrak{Q}+\mathfrak{D}$ acting in the space $\mathfrak{B}=\mathfrak{U}^{\prime}-\mathfrak{F}^{\prime}$. Since $\mathfrak{A}-\mathfrak{I}$ is isomorphic to $\mathfrak{B}$, there exists a one-to-one 1.t. $Y$ of $\mathfrak{B}$ onto $\mathfrak{A}-\Im$ such that $Y R_{x}=U_{x} Y$ for all $x \in \mathfrak{R}$, where $R_{x}$ is the mapping $u+\Im \rightarrow u x+\Im$. On the other hand, there is a one-to-one l.t. $Z$ of $\mathfrak{A}-\mathfrak{\Im}$ into $\mathfrak{H}^{\prime}-\mathfrak{Y}^{\prime}$, namely $Z: x+\mathfrak{Y} \rightarrow x+\mathfrak{Y}^{\prime}$, such that $R_{x} Z=Z T_{x}$ for all $x \in R$. Then $S=Y Z$ is a one-to-one 1.t. of $\mathfrak{B}$ into $\mathfrak{B}$ such that $U_{x} S=U_{x} Y Z=Y R_{x} Z=Y Z T_{x}=S T_{x}$ for all $x \in R$.

Finally, if $K$ has characteristic $p>0$, the degrees of the representations $U$ and $T$ are both finite, by [4, Theorem 5.1]. Proposition 4 is now completely proved.

Corollary (Zassenhaus). Let $\mathbb{R}$ be a Lie algebra over a field $K$ of characteristic $p>0$, let $D$ be a derivation of $\mathfrak{R}$, and let $x \rightarrow U_{x}$ be an irreducible representation of $\mathbb{R}$ with representation space $\mathfrak{B}$. Then there exists a representation $T$ of $\mathbb{R}$ of finite degree, a l.t. $D^{\prime}$ acting in the representation space $\mathfrak{B}$ of $T$, and a one-to-one l.t. $S$ of $\mathfrak{B}$ into $\mathfrak{B}$ such that the following conditions are satisfied: (i) $T_{x D}=\left[T_{x}, D^{\prime}\right]$ for all $x \in \mathbb{R}$; (ii) $\mathfrak{W}$ is irreducible relative to the set $T(R) \cup\left\{D^{\prime}\right\}$; and (iii) $U_{x} S=S T_{x}$ for all $x \in \mathbb{R}$.

REMARK. A necessary condition that the representation $T$ of Proposition 4 have finite degree when $K$ has characteristic zero has been obtained by Zassenhaus [11, p. 252].

Proposition 5. Let $\mathbb{R}$ be a Lie algebra over a field $K$ of characteristic $p>0$, and let $D$ be a nilpotent derivation in $L$. Let $U$ be an irreducible representation of $\mathbb{R}$, and let $f$ be an arbitrary irreducible polynomial in $K[X]$. Then there exists a representation $T$ of finite degree which satisfies conditions (i), (ii), and (iii) of the corollary to Proposition 4, and the further condition that the minimum polynomial of $D^{\prime}$ is a power of $f$.

Proof. First suppose that $\mathfrak{B} U(\mathfrak{l})=(0)$. Then $\mathfrak{B}$ is one-dimensional. If $f$ has degree $m$, then we can construct an $m$-dimensional vector space $\mathfrak{B}$ and a 1.t. $D^{\prime}$ in $\mathfrak{B}$ which has $f$ for its minimum polynomial. We define $T_{x}=0, x \in \mathbb{R}$; then $T$ and $\mathfrak{B}$ satisfy conditions (i), (ii), and (iii) of the corollary to Proposition 4 , where $S$ is any one-to-one mapping of $\mathfrak{B}$ into $\mathfrak{B}$.

Now let $\mathfrak{B} U(\mathfrak{R}) \neq(0)$. We shall adopt the notation of Proposition 4 , and in particular, we shall make use of the fact that $\mathfrak{B}$ is $\mathbb{R}$-isomorphic 
to $\mathfrak{A}-\mathfrak{Y}$, where $\mathfrak{Y}$ is a certain maximal right ideal in $\mathfrak{A}$. Let $\mathfrak{A}^{\prime}$ be the universal associative algebra of the semi-direct sum $\mathfrak{\imath}+(D)$. Since $D$ is nilpotent, some $p^{k}$ th power of it is zero, and it then follows, by a direct application of (3), that $D^{p^{k}}$ is in the center of $\mathfrak{H}^{\prime}$. We prove now that the right ideal $\mathfrak{P}=\mathfrak{F} \mathfrak{Y}^{\prime}+g(D) \mathfrak{A}^{\prime}$ is a proper right ideal in $\mathfrak{A}^{\prime}$, where $g=f^{p^{k}}$. If it is not, then since $g(D)$ is in the center, we must have a relation

$$
1=\sum_{i=0}^{r} a_{i} D^{i}+\left(\sum_{j=0}^{s} b_{j} D^{j}\right) g(D), \quad a_{i} \in \Re, b_{j} \in \mathfrak{A} .
$$

We may assume that no $b_{j} \in \mathcal{S}$, for such a term could be absorbed in the left-hand summand. Then upon comparing the coefficients of the highest power of $D$ appearing in both summands, we arrive at a contradiction.

Then $\mathfrak{B}$ can be imbedded in a maximal right ideal $\mathfrak{S}^{\prime}$, and it follows, as in the proof of Proposition 4, that $\mathfrak{S}^{\prime} \cap \mathfrak{A}=\Im$, and that the representation $T$ constructed as in Proposition 4 will satisfy the conditions of the corollary. Finally, since $\mathfrak{I}^{\prime} g(D)=g(D) \mathfrak{H}^{\prime} \subseteq \mathfrak{Y}^{\prime}, g(D)$ $\in\left(\mathfrak{\Im}^{\prime}: \mathfrak{H}^{\prime}\right)$, the annihilator of the irreducible $\mathfrak{H}^{\prime}$-module $\mathfrak{H}^{\prime}-\mathfrak{\Im}^{\prime}$. Thus the minimum polynomial of $D^{\prime}$ must divide $g=f^{p^{k}}$, and is consequently a power of $f$.

4. Irreducible representations of nilpotent Lie algebras of characteristic $p>0$. Following Zassenhaus [9], we call a basis $\left(a_{1}, \cdots, a_{n}\right)$ of a Lie algebra a regular basis if whenever $i<j, \quad\left[a_{i} a_{j}\right]$ $\in\left(a_{i+1}, \cdots, a_{n}\right)$. It is easily proved by induction on the dimension that every nilpotent Lie algebra has a regular basis.

THEOREM 2. Let $\&$ be a nilpotent Lie algebra over an arbitrary field $K$ of characteristic $p>0$, and let $\left(a_{1}, \cdots, a_{n}\right)$ be a regular basis of $\mathbb{R}$. Let $\left(f_{1}, \cdots, f_{n}\right)$ be an arbitrary ordered set of irreducible polynomials in $K[X]$. Then there exists exactly one equivalence class $\left\{U_{x}\right\}$ of irreducible representations of $\&$ such that the minimum polynomial of $U_{a_{i}}$ is a power of $f_{i}, i=1,2, \cdots, n$.

Proof. For the existence proof, we shall use induction on $n$. Suppose first that $n=1$. Then for each irreducible polynomial $f$ of degree $m$, there exists a vector space $\mathfrak{B}$ of dimension $m$, and a cyclic l.t. $A$ in $\mathfrak{B}$ whose minimum polynomial is $f$; if $\mathfrak{R}=(a)$, then $a \rightarrow A$ is an irreducible representation of $\mathcal{R}$. The uniqueness of $A$ is a known result in linear algebra.

Now assume that we have an irreducible representation $x \rightarrow U_{x}$ of $\Omega_{1}=\left(a_{2}, \cdots, a_{n}\right)$ with the required properties. Since $\left[a_{1}, \Omega_{1}\right] \subseteq \Omega_{1}$, the mapping $x \rightarrow x D=\left[a_{1}, x\right]$ is a nilpotent derivation in $\mathfrak{R}_{1}$, and ap- 
plying Proposition 5, we establish the existence of an irreducible representation $T$ such that the minimum polynomial of $T_{a_{1}}$ is a power of $f_{1}$. Let $h_{i}$ be the minimum polynomial of $T_{a_{i}}, i=2, \cdots, n$; by the corollary to Proposition 1, each $h_{i}$ is a power of an irreducible polynomial. Let $\mathfrak{B}$ and $\mathfrak{B}$ be the representation spaces of $U$ and $T$ respectively, and let $S$ be the one-to-one 1.t. of $\mathfrak{B}$ into $\mathfrak{B}$ whose existence is guaranteed by Proposition 5 . Then for each $i, 2 \leqq i \leqq n$, the restriction of $h_{i}\left(T_{a_{i}}\right)$ to the subspace $\mathfrak{B S}$ of $\mathfrak{B}$ is zero, and hence, by the induction hypothesis, the minimum polynomial of $U_{a_{i}}$, which is a power of $f_{i}$, divides $h_{i}$. Thus $h_{i}$ is a power of $f_{i}, 2 \leqq i \leqq n$, and the existence problem is settled.

We turn to the question of uniqueness. Let $U$ and $U^{\prime}$ be irreducible representations of $\mathfrak{R}$, and let $g_{x}$ and $g_{x}^{\prime}$ be the minimum polynomials of $U_{x}$ and $U_{x}^{\prime}$ respectively, for each $x \in R$. Assume further that for $i=1, \cdots, n$, the minimum polynomials $g_{a_{i}}$ and $g_{a_{i}}^{\prime}$ are both powers of the irreducible polynomial $f_{i}$. Let $\tilde{K}$ be an algebraic closure of $K$; then the extended representations $\widetilde{U}$ and $\tilde{U}^{\prime}$ split over $\tilde{K}$ as direct sums $\tilde{U}=T_{1}+\cdots+T_{s}, \tilde{U}^{\prime}=T_{1}^{\prime}+\cdots+T_{t}^{\prime}$, where $T_{i}$ and $T^{\prime}$ are the indecomposable constituents of $\tilde{U}$ and $\tilde{U}^{\prime}$ respectively. The minimum polynomials of $\tilde{U}_{x}$ and $\tilde{U}_{x}^{\prime}$ are again $g_{x}$ and $g_{x}^{\prime}$. We shall prove now that in $\tilde{K}, g_{x}$ and $g_{x}^{\prime}$ have identical sets of distinct roots. A direct calculation shows that for each $x \in L, g_{x}=\prod_{i=1}^{s} g_{i, x}$, and $g_{x}^{\prime}$ $=\prod_{i=1}^{t} g_{i, x}^{\prime}$, where $g_{i, x}$ and $g_{i, x}^{\prime}$ are the minimum polynomials ${ }^{7}$ of $T_{i, x}$ and $T_{i, x}^{\prime}$ respectively. It follows from the corollary to Proposition 1, applied to the case of an algebraically closed field, that for each $i$ and $x$, there exist elements $\alpha_{i}(x)$ and $\alpha_{i}^{\prime}(x) \in \tilde{K}$ such that $g_{i, x}(X)$ $=\left(X-\alpha_{i}(x)\right)^{e_{i}(x)}$, and $g_{i, x}^{\prime}(X)=\left(X-\alpha_{i}^{\prime}(x)\right)^{e_{i}^{\prime}(x)}$; the $\alpha_{i}(x)$ and $\alpha_{i}^{\prime}(x)$ are the characteristic roots of the l.t. $T_{i, x}$ and $T_{i, q}^{\prime}$ respectively. Our hypothesis that, for $i=1, \cdots, n$, the polynomials $g_{a_{i}}$ and $g_{a_{i}}^{\prime}$ are powers of the irreducible polynomial $f_{i}$ implies that the set of distinct characteristic roots among $\alpha_{1}\left(a_{i}\right), \cdots, \alpha_{s}\left(a_{i}\right)$ coincides with the set of distinct characteristic roots among $\alpha_{1}^{\prime}\left(a_{i}\right), \cdots, \alpha_{t}^{\prime}\left(a_{i}\right)$, for $1 \leqq i$ $\leqq n$. Zassenhaus has proved $[10$, p. 152] that the characteristic roots belonging to the elements of a regular basis, in an absolutely irreducible representation of a nilpotent Lie algebra, determine uniquely the characteristic roots of the representing 1.t. of arbitrary elements of the algebra. But the elements $\alpha_{i}(x), \alpha_{j}^{\prime}(x)$ must coincide with the characteristic roots associated with $x$ in the irreducible constituents of $T_{i}$ and $T_{j}^{\prime}$ respectively. By Zassenhaus' result, and our remark con-

7 To prove this result, we use the fact that the $g_{i, x}$ and $g_{i, x}^{\prime}$ are powers of irreducible polynomials. Then it is proved, more precisely, that $g_{x}$ and $g_{x}^{\prime}$ are the products of the distinct $g_{i, x}$ and $g_{i, x}^{\prime}$ respectively. It is this fact that is required in the proof. 
cerning the distinct characteristic roots associated with a regular basis, we conclude that the set of distinct characteristic roots among $\alpha_{1}(x), \cdots, \alpha_{s}(x)$ coincides with the set of distinct characteristic roots among $\alpha_{1}^{\prime}(x), \cdots, \alpha_{t}^{\prime}(x)$ for every $x \in \mathbb{R}$, and hence that $g_{x}$ and $g_{x}^{\prime}$ have identical sets of distinct roots in $\widetilde{K}$. This implies, as we have shown in the proof of Theorem 1 , that $U$ and $U^{\prime}$ are equivalent.

COROLlary. There exist infinitely many inequivalent irreducible representations of a nilpotent Lie algebra over an arbitrary field of characteristic $p>0$.

This result follows from Theorem 2 and the fact that if $K$ is a field, then $K[X]$ contains infinitely many distinct irreducible polynomials.

Example. As an illustration of the theory of the $u$-algebra of a restricted Lie algebra of characteristic $p>0, N$. Jacobson proved [6,

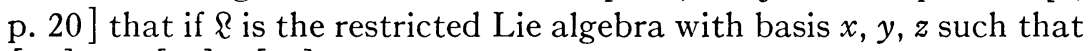
$[x y]=z,[x z]=[y z]=0, x^{p}=y^{p}=z^{p}=z$, then $\&$ has exactly $p-1$ inequivalent restricted representations. Since $\mathbb{R}$ is a nilpotent Lie algebra, we can establish this result as a consequence of Theorem 2. In fact, if $U$ is an irreducible restricted representation, then since $U_{z}^{p}$ $-U_{z}=0$, the minimum polynomial $f_{z}$ of $U_{z}$ is a prime power factor of $X^{p}-X$, hence $f_{z}(X)=X-\alpha$, where $\alpha$ is an element of the prime field. Therefore $U_{z}=\alpha I$, where $I$ is the identity l.t. If $\alpha=0$ then it is immediate that $U(\mathfrak{R})$ generates a nilpotent algebra of 1.t., and hence $U(\Omega)=(0)$. For each $\alpha \neq 0$, the minimum polynomials of $U_{x}$ and $U_{y}$ are factors of $X^{p}-\alpha$, and hence are powers of $X-\alpha^{1 / p}$. By the uniqueness part of Theorem 2 , there are exactly $p-1$ inequivalent irreducible restricted representations.

Theorem 3. Let $\mathfrak{R}, a_{1}, \cdots, a_{n} ; f_{1}, \cdots, f_{n}$ be as in Theorem 2. Then there exists a sequence $k_{i}$ of positive integers such that $\lim _{i \rightarrow \infty} k_{i}$ $=\infty$, and indecomposable representations $U^{(i)}$ of degree $k_{i}, i=1,2, \cdots$, such that for each $i$, the minimum polynomial of $U_{a_{i}}^{(i)}$ is a power of $f_{j}$, $1 \leqq j \leqq n$.

Proof. Let $U_{x}$ be the irreducible representation of $\&$ belonging to $\left(f_{1}, \cdots, f_{n}\right)$ according to Theorem 2 , and let $\mathfrak{B}$ be the kernel of the representation corresponding to $U$ of the universal associative algebra $\mathfrak{A}$ of $\mathfrak{R}$. We shall prove first that for each $i, \mathfrak{B}^{i+1}$ is properly contained in $\mathfrak{B}^{i}$. Since $\mathfrak{A}$ contains no divisors of zero, so that no finite power of $\mathfrak{B}$ is zero, it will be sufficient to prove that $\bigcap_{i=1}^{\infty} \mathfrak{B}^{i}=(0)$. As in the proof of Proposition 5, it follows that for a fixed integer $m=p^{r}, f_{1}\left(a_{1}\right)^{m}, \cdots, f_{n}\left(a_{n}\right)^{m}$ are in the center 3 of $\mathfrak{A}$, and hence the $f_{i}\left(a_{i}\right)^{m} \in \mathfrak{B}, 1 \leqq i \leqq n$. Let $\mathfrak{m}$ be the ideal in 8 generated 
by the $f_{i}\left(a_{i}\right)^{m}, 1 \leqq i \leqq n$; then $\mathfrak{m} \mathfrak{A} \subseteq \mathfrak{B}$. Suppose now that $\mathfrak{B}_{1}$ is another primitive ideal in $\mathfrak{A}$ dividing $\mathfrak{m} \mathfrak{A}$. If $U^{*}$ is the irreducible representation with kernel $\mathfrak{B}_{1}$, then since $\mathfrak{B}_{1} \supseteq \mathfrak{m} \mathfrak{A}$, the minimum polynomial of each $U_{a_{i}}^{*}$ is a power of $f_{i}, 1 \leqq i \leqq n$. By Theorem 2 , the restriction of $U^{*}$ to $\mathfrak{R}$ is equivalent to $U$, hence the kernels of the corresponding representations of $\mathfrak{A}$ coincide, and $\mathfrak{B}=\mathfrak{B}_{1}$. Therefore $\mathfrak{B}$ is the unique primitive ideal dividing $\mathfrak{m} \mathfrak{A}$, so that the radical of $\mathfrak{A} / \mathfrak{m} \mathfrak{A}$ is $\mathfrak{B} / \mathfrak{m} \mathfrak{A}$. Now we prove that $\mathfrak{A} / \mathfrak{m} \mathfrak{A}$ is finite-dimensional over $K$. Let $d_{1}, \cdots$, $d_{n}$ be the degrees of $f_{1}, \cdots, f_{n}$ respectively. Then for any standard monomial $a_{1}^{e_{1}} \cdots a_{n}^{e_{n}}$ we have

$$
a_{1}^{e_{1}} \cdots a_{n}^{e_{n}} \equiv \sum_{0 \leqq i_{j} \leqq d_{j} m, 1 \leqq j \leqq n} \xi_{i_{1}} \cdots i_{n} a_{1}^{i_{1}} \ldots a_{n}^{i_{n}}(\bmod \mathfrak{m} \mathfrak{A}),
$$

where the coefficients are in $K$. Therefore the cosets of the $a_{1}^{i_{n}} \ldots$ $a_{n}^{i_{n}} \bmod \mathfrak{m} \mathfrak{A}, 0 \leqq i_{j} \leqq d_{j} m$, form a set of generators of $\mathfrak{A} / \mathfrak{m} \mathfrak{A}$, and the dimension of $\mathfrak{A} / \mathfrak{m} \mathfrak{A}$ is finite. Then $\mathfrak{B}^{k} \subseteq \mathfrak{m} \mathfrak{A}$ for some positive integer $k$, and the intersection of the powers of $\mathfrak{B}$ will be zero if we can prove the corresponding result for $\mathfrak{m} \mathfrak{A}$. Let $\mathfrak{n}=\bigcap_{i=1}^{\infty}(\mathfrak{m} \mathfrak{Y})^{i}$. Since $\mathfrak{A}$ is a finitely generated 3 -module, where $B$ is a Noetherian ring [4], the additive theory of $\mathfrak{Z}$-submodules of $\mathfrak{A}$ due to Grundy and Snapper is applicable, and can be used to prove that $\mathfrak{m} \mathfrak{n}=\mathfrak{n}[5]$. It then follows, exactly as in the commutative case, that since $\mathfrak{A}$ contains no divisors of zero, $\mathfrak{n}=0.8$

Now we have established that $\mathfrak{B} \supset \mathfrak{B}^{2} \supset \cdots$, and on the other hand, each $\mathfrak{A} / \mathfrak{B}^{r}$ is a finite-dimensional algebra [11, p. 263]. Since $\mathfrak{A} / \mathfrak{B} \cong\left(\mathfrak{A} / \mathfrak{B}^{r}\right) /\left(\mathfrak{B} / \mathfrak{B}^{r}\right), r=1,2, \cdots, \mathfrak{A} / \mathfrak{B}^{r}$ is a primary algebra with simple residue class algebra $\mathfrak{A} / \mathfrak{B}$. If $h_{i}$ is the dimension of $\mathfrak{A} / \mathfrak{B}^{i}$ over $K$, then the fact that the powers of $\mathfrak{B}$ form a properly decreasing sequence implies that $\lim _{i \rightarrow \infty} h_{i}=\infty$. Let $\Im_{i}$ be an indecomposable right ideal in $\mathfrak{A} / \mathfrak{B}^{i}, i=1,2, \cdots$; if $k_{i}$ is the dimension of $\Im_{i}$ over $K$, then since $h_{i} / k_{i}$ is equal to the number of minimal right ideals in a direct decomposition of $\mathfrak{A} / \mathfrak{B},{ }^{9} \lim _{i \rightarrow \infty} k_{i}=\infty$. Then the representation of $\mathfrak{A}$ given by $R_{x \nu_{i}}$, where $\nu_{i}$ is the natural homomorphism of $\mathfrak{A} \rightarrow \mathfrak{U} / \mathfrak{B}^{i}$, and $R_{x \nu_{i}}$ is the mapping $u \rightarrow u\left(x \nu_{i}\right)$ in $\Im_{i}$, defines an indecomposable representation of $\mathfrak{A}$ of degree $k_{i}$. Finally we note that since $\nu_{i}$ annihilates some power of each $f_{i}\left(a_{i}\right), 1 \leqq i \leqq n$, it follows that the minimum polynomial of $R_{a_{i} \nu_{i}}$ is a power of $f_{j}, 1 \leqq j \leqq n$, and $i=1,2, \cdots$. The representations $U^{(i)}$ required by the statement of the theorem are then simply the restrictions of $R_{x v_{i}}$ to $\mathfrak{R}$, and the theorem is proved.

${ }^{8}$ Cf. A. Weil, Foundations of algebraic geometry, New York, 1946, p. 45.

${ }^{\circ} \mathrm{Cf}$. E. Artin, C. Nesbitt, and R. Thrall, Rings with minimum condition, Ann Arbor, 1945, Chapter 9. 


\section{REFERENCES}

1. G. Birkhoff, Representability of Lie algebras and Lie groups by matrices, Ann. of Math. vol. 38 (1937) pp. 326-332.

2. C. Chevalley, Thérie des groupes de Lie, vol. II, Paris, 1951.

3. - Thérie des groupes de Lie, vol. III, to appear.

4. C. W. Curtis, Noncommutative extensions of Hilbert rings, Proc. Amer. Math. Soc. vol. 4 (1953) pp. 945-955.

5. - On the structure of non-semisimple algebras, Duke Math. J. vol. 21 (1954) pp. 79-86.

6. N. Jacobson, Restricted Lie algebras of characteristic p, Trans. Amer. Math. Soc. vol. 50 (1941) pp. 15-25.

7. - Un gênêralisation du Théorème d'Engel, C. R. Acad. Sci. Paris vol. 234 (1952) pp. 679-681.

8. E. Witt, Treue Darstellung Liescher Ringe, J. Reine Angew. Math. vol. 176 (1937) pp. 126-140.

9. H. Zassenhaus, Über Liesche Ringe mit Primzahlcharacteristik, Abh. Math. Sem. Hansischen Univ. vol. 13 (1940) pp. 1-100.

10. - Darstellungstheorie nilpotenter Lie-ringe bei Characteristik $p>0$, J. Reine Angew. Math. vol. 182 (1940) pp. 150-155.

11. - Über die Darstellungen der Lie-algebren bei Characteristik 0, Comment. Math. Helv. vol. 26 (1952) pp. 252-274.

UNIVERSITY OF Wisconsin 